\title{
Self Stabilization of Dynamic Systems
}

\section{Assuming Only Read/Write Atomicity}

June 9,1992

Shlomi Dolev ${ }^{\prime}$

Amos Israelif

Shlomo Moran $\dagger$

\begin{abstract}
Three self-stabilizing protocols for distributed systems in the shared memory model are presented. The first protocol is a mutual exclusion protocol for tree structured systems. The second protocol is a spanning tree protocol for systems with any connected communication graph. The third protocol is a self stabilizing protocol for mutual exclusion, for systems with a general (connected) communication graph. This last protocol is obtained by combining the previous two protocols. The combination employs a simple technique called fair protocol combination, which is enabled by both the self-stability and by the flexibility of dynamic protocols. The presented protocols improve upon previous protocols in two ways: First, it is assumed that the only atomic operations are either read or write to the shared memory. Second, our protocols work for any connected network and even for dynamic networks, in which the topology of the network may change during the execution.
\end{abstract}

$†$ Department of Computer Science, Technion - Israel Institute of Technology, Haifa 32000, ISRAEL. Part of this research was supported in part by Technion V.P.R. Funds - Wellner Research Fund, and by the Foundation for Research in Electronics, Computers and Communications, administrated by the Israel Academy of Sciences and Humanities.

¥ Department of Electrical Engineering, Technion - Israel Institute of Technology, Haifa 32000, ISRAEL. 


\section{Introduction}

A self-stabilizing system which is started from an arbitrary initial configuration, regains its consistency and demonstrates legal behavior by itself, without any kind of an outside intervention. Consequently, a self-stabilizing system need not be initialized to any particular configuration, and can recover from transient bugs, bugs which change the state of one or more components of the system but keep those components in working order. In this paper we present self-stabilizing protocols for mutual-exclusion and for constructing a spanning tree. The presented protocols work on connected networks of arbitrary topology which can change dynamically during execution. Communication among neighboring processors is carried out by use of communication registers (called registers throughout this paper). The atomic operations that these registers support are read and write.

We model distributed self-stabilizing systems as a set of state machines called processors. Each processor can communicate with some subset of the processors called its neighbors. The system's communication graph is the graph formed by representing each processor as a node and by drawing an edge between every two neighbors. A protocol is a parameterized family of systems where the parameters can vary over the number of different state machines used by the protocol, the various families of communication graphs, the set of atomic operations supported by the communication registers, etc. A processor's degree is equal to the number of its neighbors. A protocol is uniform if all processors of the same degree are identical. If all processors of the same degree are identical except a single processor in the entire system, then the protocol is semi-uniform. An atomic step is the "largest" step that is guaranteed to be executed uninterruptedly. A protocol uses composite atomicity if some atomic step contains (at least) a read operation and a write operation. A processor uses read/write atomicity if each atomic step contains either a single read operation or a single write operation but not both. The behavior of the system is modeled by the interleaving model in which processors are activated by a scheduler. Whenever an enabled processor is activated, it executes a single atomic step. To ensure the correctness of a protocol, the scheduler is regarded as an adversary and the protocol is required to be correct in all possible executions. The common schedulers are the central demon which activates processors one by one and the distributed demon which activates subsets of processors.

The class of self-stabilizing protocols was defined by Dijkstra in his pioneering paper [Dij74]. In this paper Dijkstra presents three semi-uniform, self-stabilizing, mutual-exclusion protocols for rings. Protocols in the same setup but under the distributed demon are presented by Brown, Gouda and Wu in [BGW87], and by Burns in [Bu87]. Burns and Pachl in [BP87] present a uniform, self-stabilizing, mutual-exclusion protocol for rings with a prime 
number of processors. A semi-uniform, self-stabilizing protocol for some variant of the mutual-exclusion problem which runs on tree systems is presented by Kruijer in [Kr79]. A self-stabilizing, mutual-exclusion protocol for systems with arbitrary communication graphs is presented by Tchuente in [Tc81]. Unlike the aforementioned protocols the protocol presented in [Tc81] is not semi-uniform, in fact the program of each processor depends on the system's communication graph, for many communication graphs all processors are distinct. Furthermore, obtaining the protocol for each individual system requires an extensive programming work.

All previous self-stabilizing protocols use composite atomicity. In the work of Loui and Abu-Amara in [LA87], it was shown that while there exists no consensus protocol for systems that use read/write atomicity, the consensus task is solvable for systems that use composite atomicity. Since any system under composite atomicity can trivially emulate an equivalent system that uses read/write atomicity, composite atomicity is strictly stronger than $\mathrm{read} / \mathrm{write}$ atomicity.

A protocol is dynamic if it tolerates changes in the communication graph during execution as long as the communication graph remains connected. The changes we allow are processor addition or removal and link addition and removal. Every self-stabilizing, uniform protocol that works on every communication graph is dynamic, since it stabilizes after any topology change. A semi-uniform protocol that works on any communication graph is dynamic as long as the (single) special processor is not removed from the system. In [Dij74], Dijkstra used symmetry considerations and showed that for rings of composite size, there exists no uniform, self-stabilizing, mutual-exclusion protocol. Thus if one opts for dynamic, self-stabilizing, mutual-exclusion protocols then the best that can be achieved are semi-uniform protocols.

Previous works assumed that one way communication from $P_{1}$ to $P_{2}$ is carried out by $P_{1}$ changing its state which is observable by $P_{2}$. This mode of communication is equivalent to the use of a single communication register in which $P_{1}$ writes and from which all processors to which it can communicate read. It is not hard to show that under this communication mode, there exists no semi-uniform, self-stabilizing, mutual-exclusion protocol in many systems, including systems with very simple communication graphs. There are two possible ways to remedy this problem: The first one, which was chosen by Tchuente in [Tc-81], is to give up uniformity altogether and program each processor individually. Since in this method each processor is programmed individually, it cannot yield dynamic protocols. The alternative way, which we choose in this work, is to allow each processor to break the symmetry among its neighbors locally. This is done by introducing a link between every pair of neighbors. Each link is composed of two 
registers and supports two-way communication. One neighbor writes in the first register and reads from the second, the other neighbor reads from the second register and writes in the first. Each register is serializable (atomic) with respect to read and write actions.

We present two semi-uniform, self-stabilizing protocols: The first protocol is a mutual-exclusion protocol for tree structured systems. The second protocol constructs a spanning tree of the system's communication graph; both protocols are correct under read/write atomicity. We then present fair protocol combination as a technique for combining self-stabilizing protocols into another self-stabilizing protocol. The presentation is completed by combining the two aforementioned protocols into a semi-uniform, self-stabilizing, mutual-exclusion protocol for systems with any connected communication graph using fair protocol combination. The combined protocol, like both its building blocks, is correct under read/write atomicity. Using this final protocol we show that any protocol which is selfstabilizing under composite atomicity can be executed in a self-stabilizing fashion in the presence of read/write atomicity.

Our protocols improve upon all previous protocols in two important aspects:

- Atomicity: All previous self-stabilizing protocols use composite atomicity. Our protocols use read/write atomicity, hence they subsume all aforementioned self-stabilizing protocols.

- Topology: Almost all previous self-stabilizing protocols work only on restricted families of communication graphs. In this respect our protocols improve upon all previous protocols except the protocol of [Tc81], since they work in systems with arbitrary connected communication graphs. Furthermore, our protocols are semiuniform hence they are also dynamic and superior to the protocol of [Tc81].

The rest of this paper is organized as follows: in Section 2 the computational model and the requirements for self-stabilization are discussed and formally defined. In Section 3 we present a simple self-stabilizing protocol called the balance-unbalance protocol for mutual-exclusion in a two processor system and show how to adapt it to read/write atomicity. In Section 4 we present a self-stabilizing mutual-exclusion protocol for tree-structured systems which uses the balance-unbalance protocol as a building block. In Section 5 we present fair protocol combination, then we proceed to present a self-stabilizing protocol for finding a spanning tree of the system's communication graph. Combining the spanning tree protocol with the mutual-exclusion protocol yields the final protocol. Concluding remarks are brought in Section 6. 


\section{Model and Requirements}

2.1. The Model. A distributed system consists of $n$ processors, denoted by $P_{1}, P_{2}, \cdots, P_{n}$. Each processor is a (possibly infinite) state machine. Processor $P_{1}$ is called special. All other processors are called normal. Normal processors have no distinct identities, the subscripts $2, \cdots, n$ are used for ease of notation only. Two normal processors with the same number of neighbors are identical. Neighbors, $P_{i}$ and $P_{j}$ communicate with each other by using two shared registers, $r_{i j}$ in which $P_{i}$ writes and from which $P_{j}$ reads and $r_{j i}$ in which $P_{j}$ writes and from which $P_{i}$ reads. All links incident to each processor $P_{i}$ are ordered by some arbitrary ordering $\alpha_{i}$ which induces in a natural way an ordering of the neighbors of $P_{i}$. The collection of all these orderings is denoted by $\alpha=\left(\alpha_{1}, \cdots, \alpha_{n}\right)$.

Every register $r$ is associated with the set $\Sigma_{r}$ of permitted values which can be stored in $r$ (the set $\Sigma_{r}$ is not necessarily finite). Each register $r$ has a writer - a processor that can write in $r$, and a reader - a processor that can read from $r$. A write operation to $r$ stores a value from $\Sigma_{r}$ in $r$. A read operation retrieves the value (from $\Sigma_{r}$ ) stored in $r$. Each register is serializable with respect to read and write operations. The registers in which processor $P$ can write are called the registers of $P$. We choose to look at a processor and its registers as a single entity, thus the state of a processor fully describes the values stored in its registers. Denote by $S_{i}$ the set of states of $P_{i}$. A configuration of the system is the vector of states of all processors. Denote by C $=\left(S_{1} \times S_{2} \times \cdots S_{n}\right)$ the set of all possible configurations of the system.

An atomic step of a processor consists of an internal computation followed by a read or a write operation, but not both. Processor activity is managed by a scheduler, which is also called the central demon. In any given configuration the demon activates a single processor which executes a single atomic step. An execution $E$, of the system, is an infinite sequence of configurations $E=C_{1}, C_{2}, \cdots$ where for every $i>0, C_{i+1}$ is reached from $C_{i}$ by a single atomic step of a single processor. An infinite execution is fair if every processor executes steps infinitely often.

2.2. Task Specification and Self-Stabilizing Protocols. A self-stabilizing system demonstrates legitimate behavior some time after it is started from an arbitrary configuration. A natural way to specify a behavior in an abstract way is by a set of sequences of configurations. We define tasks as sets of legitimate-sequences. The semantics of any specific task is expressed by requirements on its sequences. Intuitively each legitimate sequence can be thought of as an execution of a protocol but we do not require it formally. For instance, the mutual exclusion task is 
defined as the set of sequences of configurations which satisfy: Each processor has a subset of its states called critical section; in each configuration, at most one processor is in its critical section, and every processor is in its critical section in infinitely many configurations.

To formally define a task $T$, one should specify for each possible system $S T$, a set of legitimate sequences for $S T$. The task $T$ is defined as the union of the legitimate sequence set over all possible systems. A configuration $C$ of a system is safe with respect to a task $T$ and a protocol $\operatorname{Pr}$ if any fair execution of $\operatorname{Pr}$ starting from $C$ belongs to $T$. A protocol and a scheduler determine the set of all possible executions of the protocol under this scheduler. In the nonself-stabilizing model, a protocol implements a task if all its executions belong to the set of sequences which constitutes the task. In the self-stabilizing model this requirement is relaxed, and the protocol is self-stabilizing for the task if in each possible execution it eventually reaches a safe configuration. Hence we define a protocol to be selfstabilizing with respect to a task $T$ if the following definition holds:

\section{[Self Stabilization]}

A protocol is self-stabilizing if starting from any system configuration, it eventually reaches a safe configuration.

This definition separates the specific task which the protocol implements from the general requirements for self-stabilization and allows self-stabilizing protocols for any task. It is natural (though not necessary) to require that a task is closed under the suffix operation. When this requirement is adopted, any configuration which is reachable from a safe configuration is also safe, therefore the set of safe configurations for task $T$ with respect to protocol $\operatorname{Pr}$ is closed under executions of $\mathrm{Pr}$.

2.3. Protocol Description. A protocol is specified by describing two types of processors: A special processor and a normal processor. A processor is entirely determined by its type and by the number of its neighbors. For convenience we choose to represent each of our processors as a $R A M$ executing a program. Since the system is dynamic the number of neighbors of each processor may change during execution. This is modeled by assuming that each processor has access to a local constant called no_neighbors in which the number of the processor's neighbors is stored. This constant is assumed to be updated by the hardware whenever the number of neighbors is changed. (Later we discuss the technique of protocol combination. In a combined protocol no_neighbors can be updated by a lower level protocol) 
The program of each processor is partitioned to distinct atomic steps. It is assumed that each program is executed step by step where each step is executed uninterruptedly. Each processor is assumed to be equipped with a program counter $(p c)$ whose value indicates the next atomic step to be executed. The partition of the program into atomic steps is straight forward: Each atomic step consists of a sequence of internal operations which ends either with a write operation or with a read operation. The state of a processor is determined by the internal state of the $R A M$ and the contents of its registers. The internal state of the $R A M$ is fully described by the values stored in its internal variables and by its next step (the $p c$ ). Each internal variable has a set of permitted values, a permitted state of a processor is any assignment of permitted values to its internal variables and to its registers.

\section{The Balance-Unbalance Protocol}

3.1. The Basic Protocol. A processor is enabled if it can execute a state-transition. A mutual-exclusion protocol under composite atomicity is designed such that in each legitimate configuration there exists a single enabled processor. The enabled processor is privileged, it has the right to enter its critical section. Presumably the enabled processor finds out that it is enabled by reading its neighbors' registers, executes its critical section, and then passes the privilege to one of its neighbors by executing a state transition which includes writing new values in some of its registers. The composite atomicity ensures that this extended atomic step is executed uninterruptedly. The balanceunbalance protocol is probably the simplest protocol for mutual-exclusion under composite atomicity. It is designed for a system of two processors, which are connected by a link. The two processors are the unbalancing processor $U B$ and the balancing processor $B A$, Each processor has two states, denoted by 0 and 1 . The configuration of a system is defined by the states $\left(s_{1}, s_{2}\right)$ of $U B$ and $B A$ respectively. Thus, the system has four possible configurations: $(0,0),(1,0),(1,1)$, and $(0,1)$. Processor $U B$ is enabled when the link is balanced. Its transition function unbalances the link by transfering $(0,0)$ to $(1,0)$ and $(1,1)$ to $(0,1)$. Analogously, $B A$ is enabled when the link is unbalanced. Its transition function balances the link by transfering $(0,1)$ to $(0,0)$ and $(1,0)$ to $(1,1)$.

Consider an execution of the protocol under composite atomicity. In any possible configuration, exactly one processor is enabled (and privileged); the enabled processor passes the privilege to the other processor by changing its state. Thus, starting with any configuration of the system, and regardless of the specific behavior of the demon, the system configuration is changed repeatedly according to the following cycle: $((0,0),[U B$ writes], $(1,0),[B A$ writes], $(1,1),[U B$ writes], $(0,1),[B A$ writes $],(0,0))$. Therefore, this protocol is a self-stabilizing, mutual-exclusion 
protocol in the strongest possible sense: There is a unique legitimate sequence of configurations, which is a suffix of every possible execution of the protocol. In a way, this protocol is well known and is a simplified version of protocols presented in [Dij74], [PF77], [Kr79], and [Bu87].

Under read/write atomicity an atomic step includes either a read action or a write action. For convenience we place this single action by the end of the step including it. When an atomic step ends by a read action, the read value may affect the next transition. Therefore the state of each processor should reflect the last value it read from the (register of the) other processor. We use here the term state to denote the full information describing the processor behavior, while the balance-unbalanced bits are called colors. The state of $U B$ is described by the following components: $\{$ the color of $U B$, the last color of $B A$ read by $U B$, the next action to be executed by $U B\}$. The state of $B A$ is described analogously as follows: \{the last color of $U B$ read by $B A$, the color of $B A$, the next action to be executed by $B A$ \}. (for better understanding, the two components of $B A$ are switched) A configuration of the system is a pair of the processor states.

Lemma 3.1: Under read/write atomicity the balance-unbalance protocol is not a self-stabilizing mutual-exclusion protocol.

proof: Consider the configuration ( $\{0,0$, write $\}\{1,0$,write $\})$, in which the colors of $U B$ and $B A$ are both 0 , but as a result of a transient bug, $B A$ "thinks" that the color of $U B$ is 1 . In this configuration both processors are enabled (and hence privileged).

$(\{0,0$, write $\}\{1,0$, write $\})[B A$ writes $]$,

$(\{0,0$, write $\}\{1,1$, read $\})[B A$ reads $]$,

( $\{0,0$, write $\}\{0,1$, write $\})[U B$ writes $]$,

$(\{1,0$, read $\}\{0,1$, write $\})[U B$ reads $]$,

$(\{1,1$,write $\}\{0,1$, write $\})[B A$ writes $]$,

$(\{1,1$, write $\}\{0,0$, read $\})[B A$ reads $]$,

$(\{1,1$,write $\}\{1,0$,write $\})[U B$ writes $]$,

$(\{0,1$, read $\}\{1,0$, write $\})[U B$ reads $]$,

$(\{0,0$, write $\}\{1,0$, write $\})[B A$ writes $]$,

Figure 3.1: A Prefix of a Non-Stabilizing Execution 
In Figure 3.1 we bring a prefix of an execution, starting and ending with the above configuration. In this prefix each processors is activated and both processors are simultaneously privileged. Since this prefix starts and terminates with the same configuration it can be duplicated infinitely often to obtain an infinite fair execution. In half of the configurations of this infinite execution $U B$ and $B A$ are both privileged, hence the system does not stabilize.

3.2. Adaptation for Read/Write Atomicity. In this section we modify the balance-unbalance protocol to be correct under read/write atomicity. The modification implements several ideas which are helpful in general for selfstabilizing protocols under read/write atomicity. The code for the modified protocol appears in Figure 3.2. The registers of $U B$ and $B A$ are called $r_{u b}$ and $r_{b a}$ respectively. Processor $U B$ has two internal variable called $m y \_c o l o r$ and $b a_{-}$color. These variable constitute the view of $U B$, the values it "thinks" $r_{u b}$ and $r_{b a}$ have. In case the colors of $r_{u b}$ and $r_{b a}$ are equal to the values of variables my_color and ba_color respectively, we say that $u b$ has a correct view. Analogously, processor $B A$ has two internal variables called $m y \_c o l o r$ and $u b \_c o l o r$ which constitute $B A$ 's view. In case the colors of $r_{b a}$ and $r_{u b}$ are equal to the values of $m y_{-}$color and $u b_{-}$color respectively, we say that $B A$ has a correct view.

The program for each processor in the modified protocol consists of a loop which is executed repeatedly. The loops for both processors have a similar structure. Each loop consists of two blocks: a refresh block and a main block. In the refresh block each processor unconditionally copies its internal variable my_color to its register. In this way the processor ensures that the color of its register is equal to the processor's "belief". The unconditional write is called the refreshing write. Following the first refreshing write the value of the register can always be inferred from the values of the processor's internal variables, hence the only refreshing write which may change the value of a register is the first write in an execution.

After refreshing its register each processor proceeds to its main block which includes the processor's critical section. Unlike the refresh block, execution of the main block is conditional. The first atomic step in the main block is a read action. The value read in this action is used as a guard for the rest of the main block in which the critical section is executed. The problem depicted in Figure 3.1 is caused by the nature of read/write atomicity. A processor may read the color of the other processor, then the second processor may write and change its color. After that, the first processor may use the color it read, which is already outdated at this point, and enter its critical section. Consequently mutual-exclusion might be violated. In order to overcome this problem, some additional synchronization 
$U B$ : repeat forever $r_{u b}:=$ write $($ my_color, 0$)$

$B A$ : repeat forever

$r_{b a}:=\operatorname{write}($ my_color $)$

repeat $\left(u b \_c o l o r, c l o s e\right):=\operatorname{read}\left(r_{u b}\right)$

until close $=0$

if $u b \_$color $\neq m y \_c o l o r$

then begin

CRITICAL SECTION

my_color $:=u b \_c o l o r$

end refresh

link seems balanced

main loop

close link

reread ba_color

complement your color

unbalance and open link

refresh

link seems open and unbalanced main loop

complement ba_color

balance link

Figure 3.2: The Modified Balance-Unbalance Protocol

between the processors is required. For this purpose $U B$ is allowed to close the link for $B A$ using the binary close field with which $r_{u b}$ is augmented. Using the close field $U B$ controls the number of times $B A$ executes its main block between every consecutive executions of $U B$ 's main block. Eventually the system is stabilized as proved below.

A system configuration is specified by the values of the variables my_color (of both processors), the values of ba_color and $u b \_c o l o r$, the values of the registers and the next step each processor is about to execute (the $p c$ of both processors). After both registers are refreshed the values of the my_color variables are the same as the values of the corresponding registers. In this situation and when the values of the $p c$-s are implied by the context, we describe a configuration by a 5-tuple (ba_color, $r_{u b}$.color, $r_{u b}$.close $\left.\rightarrow r_{b a}, u b \_c o l o r\right)$ which is called the link descriptor. The arrow in the link descriptor stands for the link that separates the variables and register values of $U B$ (at the tail) from those of $B A$.

Under composite atomicity a non-enabled processor which is activated by the demon does not execute any state transition. The situation is different under read/write atomicity where a processor can always execute some action. This action may be a read action after which the processor may find that it cannot execute any write action. Another possibility is that the processor finds out that it can execute a write action, but the written value is equal to the value which is stored in the register before the write action is executed. In order to accommodate these situations 
we define some segment of a processor's execution as a stuttering segment if the only changes in the processor's state are in its $p c$. A stuttering section that starts and ends with the same system configuration is called a $c y c l i c$ stuttering section. A cyclic stuttering section might be removed from an execution, except the first (or last) configuration, and the resulting sequence is also an executions. We say that two executions are equivalent up to stuttering if when all cyclic stuttering sections are removed the resulting system executions are equal.

Consider a configuration $C$ in which the registers are refreshed, the value of $r_{u b}$ is $(0,0)$ and the value of $r_{b a}$ is 0 . The link descriptor of $C$ is $(0,0,0 \rightarrow 0,0)$. In such a configuration, the only possible subsequent changes in the value of the link descriptor are given by the legitimate cycle which appears in Figure 3.3: (In the legitimate cycle the refreshing writes are omitted)

$$
\begin{aligned}
& (0,0,0 \rightarrow 0,0) \\
& \text { [UB writes], }(0,0,1 \rightarrow 0,0) \text {, } \\
& {[U B \text { reads], }(0,0,1 \rightarrow 0,0) \text {, }} \\
& \text { [ } U B \text { writes], }(0,1,0 \rightarrow 0,0) \text {, } \\
& {[B A \text { reads }],(0,1,0 \rightarrow 0,1) \text {, }} \\
& \text { [BA writes], }(0,1,0 \rightarrow 1,1) \text {, } \\
& {[U B \text { reads], }(1,1,0 \rightarrow 1,1) \text {, }} \\
& \text { [UB writes], }(1,1,1 \rightarrow 1,1) \text {, } \\
& \text { [ } U B \text { reads], }(1,1,1 \rightarrow 1,1) \text {, } \\
& \text { [UB writes], }(1,0,0 \rightarrow 1,1) \text {, } \\
& \text { [BA reads],(1, 0, } 0 \rightarrow 1,0) \text {, } \\
& \text { [ } B A \text { writes], }(1,0,0 \rightarrow 0,0) \text {, } \\
& \text {,[UB reads], }(0,0,0 \rightarrow 0,0) \text {, }
\end{aligned}
$$

Figure 3.3: The legitimate cycle

Define $B U B^{\prime}$ to be the set containing the sequence $l$ obtained by a repeated execution of the legitimate cycle and all suffixes of $l$. The task $B U B$ is now defined as the set of all sequences which are equivalent to some task in $B U B^{\prime}$ up to stuttering. Note that $B U B$ is a subtask of the mutual-exclusion task. By this definition, each configuration in the legitimate cycle is safe for the set $B U B$. Observe that when the system is in the legitimate cycle the processors access their critical section in a mutually exclusive (and fair) fashion. In the following lemmas we prove that the protocol is self-stabilizing by showing that in every fair execution some configuration in the legitimate cycle is reached.

Lemma 3.2: In every fair execution $E$ of the protocol in which the color of no register is changed, there is a configuration $C_{t}$ after which the link is always open. 
proof: Execution $E$ is fair, therefore $U B$ is activated infinitely often, in particular $r_{u b}$ is refreshed infinitely often. Consider configuration $C_{t}$ right after $r_{u b}$ is refreshed for the first time. In $C_{t}$ the link is open since it was opened in the refreshing write of $U B$. In its next activation $U B$ reads $r_{b a}$; since during $E$ the color of no register changes, after this read action $U B$ has a correct view which is constant throughout $E$. In case the link is unbalanced in this constant view, $U B$ does not enter its loop and therefore never closes the link. In case the link is balanced in the constant view, $U B$ executes the loop and unbalances the link, contradiction to the assumption that no color is changed during $E$.

Lemma 3.3: In every fair execution of the protocol both registers are refreshed infinitely often.

Proof: The Lemma holds trivially for $r_{u b}$. Assume towards a contradiction that $E$ is a fair execution in which $r_{b a}$ is never refreshed. This is possible only if whenever BA executes step 7, it finds that the link is closed. By Lemma 3.2 this implies that the content of some register is changed infinitely often. If register $r_{b a}$ is changed infinitely often then we are done, so it must be the case that only the content of register $r_{u b}$ is changed infinitely often. In particular, it implies that there is a suffix $E^{\prime}$ of $E$ in which the content of $r_{u b}$ is changed infinitely often, but the content of $r_{b a}$ is never changed. We complete the proof by showing that this latter scenario is impossible.

Consider two successive changes of $r_{u b}$ in $E^{\prime}$, which are done after $r_{u b}$ is refreshed. The first change is done after $U B$ executes step 4, and learns that the link is balanced. Since $B A$ never writes in $E^{\prime}$, after $U B$ writes (in step 5), the link becomes unbalanced. The next time $U B$ executes step 2 it finds that the link is unbalanced, and hence it does not change the value of $r_{u b}$ anymore - a contradiction.

Corollary 3.4: In every fair execution $E, U B$ closes the link, by executing step 3, infinitely often.

Proof: Let $E^{\prime}$ be a suffix of $E$ in which both registers are refreshed, as guaranteed by Lemma 3.3. Assume for contradiction that the link is never closed during $E^{\prime}$. In particular this implies that $U B$ never changes the color of $r_{u b}$ during $E^{\prime}$, and also that infinitely often during $E^{\prime}$, the color of $r_{b a}$ is not equal to the (constant) color of $r_{u b}$. This implies that eventually, $B A$ balances the link by changing the color of $r_{b a}$. Once $B A$ have done this, it does not change the color again, unless the link becomes unbalanced. This means that the next time $U B$ executes step 2 , it will find out that the link is balanced, and hence it executes step 3 and closes the link - a contradiction.

Lemma 3.5: In every fair execution $E$ of the protocol, the system reaches a configuration in the legitimate cycle.

Proof: By Lemmas 3.3 and 3.4, there is a suffix $E^{\prime}$ of $E$ during which both processors are refreshed, and during $E^{\prime}$ $U B$ closes the link. After closing the link, $U B$ reads the value of $r_{b a}$, changes the color of $r_{u b}$ (if necessary), and 
opens $r_{u b}$ for reading. Call the sequence of configurations during which $U B$ executes these operations the close period of $r_{u b}$. Consider the behavior of the link during the close period of $r_{u b}$. Whenever $B A$ reads $r_{u b}$ during the close period, it repeats executing line 7 until $r_{u b}$ is opened. Therefore $B A$ can change the color of $r_{b a}$ (by executing step 8) at most once, during the close period, since after any such change, $B A$ reads $r_{u b}$. During the close period $U B$ reads $r_{b a}$ once, the following three cases sum up the possible ways in which the link descriptor might be changed by $B A$, during the close period of $r_{u b}$ :

Case 1: $B A$ does not change $r_{b a}$ during the close period.

Case 2: $B A$ changes $r_{b a}$ before $U B$ reads from it.

Case 3: $B A$ changes $r_{b a}$ after $U B$ reads from it.

In the first two cases $U B$ reads the updated value of $r_{b a}$ and unbalances the link (if it is not already unbalanced) hence the link descriptor in the configuration that immediately follows the close period is equal to either $(1,0,0 \rightarrow 1$, ?) or $(0,1,0 \rightarrow 0, ?)$ (the question mark stands for either 0 or 1$)$, which are all in the legitimate cycle. The third case starts as follows: $U B$ reads $r_{b a}$, then $B A$ changes the color of $r_{b a}$ to be equal to $u b_{-}$color. At this stage the link descriptor is either $(0, ?, 1 \rightarrow 1,1)$ or $(1, ?, 1 \rightarrow 0,0)$. At the end of the close period, $U B$ tries to unbalance the link using the last (not updated) color it read from $r_{b a}$. Thus the value of the link descriptor in the configuration that immediately follows the close period is either $(0,1,0 \rightarrow 1,1)$ or $(1,0,0 \rightarrow 0,0)$ which are both in the legitimate cycle.

\section{A Mutual-Exclusion Protocol for Dynamic Tree Systems}

In this section we present a self-stabilizing, mutual-exclusion protocol for systems whose communication graph is a directed tree, rooted at the (special) root processor. The protocol is dynamic as long as the topology changes preserve the tree structure. The tool by which the privilege is passed along links is the balance-unbalance protocol. Each link $e$, is regarded as directed from $U B$ to $B A$. The registers of $e$ are called the unbalance register of $e$ and the balance register of $e$, respectively. Thus in the tree protocol a processor with no_sons sons plays the role of UB no_sons times, and each normal processor plays the role of $B A$ once. A processor is privileged in the tree protocol if it is privileged in all the balance-unbalance protocols in which it participates, that is when all its outgoing links are balanced and its incoming link (for a non-root processor) is unbalanced. 
After stabilizing, an execution of the protocol proceeds in phases. Execution of a phase corresponds to a $D F S$ tour of the whole tree, where the DFS's " center of action" is at the node of the privileged processor. The first privileged processor in each phase is the root. Following its first activation the root (recursively) passes the privilege to (the subtrees rooted at) its sons in a left to right order. A processor $P$ that becomes privileged for the first time in some phase, passes the privilege to its leftmost son. Once the privilege is passed to all processors in the subtree rooted at this son it is returned to $P$. Subsequently the privilege is passed to $P$ 's second son from the left, and so on. The phase ends when the rightmost son of the root returns the privilege to the root itself. In each phase the privilege is passed twice along each edge, once in each direction. Consequently, each processor becomes privileged $d$ times where $d$ is the processor's degree.

At the beginning of each phase all registers in the tree are colored by one color (say 0), during the phase the tree is recolored by the complementing color (in this case 1). For each edge $e$, its unbalance register is recolored whenever the privilege is passed through $e$, and its balance register is recolored when the privilege is returned back through $e$. Thus in every intermediate configuration the tree is partitioned by a path of unbalanced edges that goes from the root to the DFS's "center of action", which is the node of the privileged processor. Every unbalance register on this path is colored with the new color (1), while all balance registers on this path are colored with the old color (0). All edges left of this path are colored with the new color (1) while all edges right of the path are colored by the old color (0).

Each processor $P$ has two internal variables called new_color and border, where new_color is binary and border ranges between 0 and no_sons. The value of new_color indicates the color in which the tree is recolored, the value of border indicates the border between outgoing links of $P$ which are already recolored with new_color and those whose color is yet to be changed along the phase. In addition each processor has the internal variables needed for all instances of the balance-unbalance protocol in which it participates. The state set of $P_{i}$ is $S_{i}$. The set $S_{i}$ contains every possible assignment of permitted values to the variables of $P_{i}$. The set $C$ of system configurations consists of all possible combinations of processor states and register values.

The protocol for the root and for a normal processor is written for processor $P$ with no_sons sons. The unbalance register of $P$ 's incoming link (which is written by $P$ 's father and which is read by $P$ ) is denoted by $q_{u b}$, the balance register of $P$ 's incoming link (which is written by $P$ and which is read by $P$ 's father) is denoted by $r_{b a}$. The registers of $P$ 's outgoing links are denoted as follows: for each outgoing edge $e_{m}, 1 \leq m \leq$ no_sons, the unbalance 
register of $e_{m}$, (which is written by $P$ and which are read by $P$ 's son) is denoted by $r_{u b}[m]$ and the balance register of $e_{m}$ (which is written by $P$ 's son and which is read by $P$ ) is denoted by $s_{b a}[m]$. A picture of a node and the registers on its links appear in Figure 4.1 .

Figure 4.1: A pictorial description of a node in the system. 
Root: repeat forever

1

2

3

4

5

6

Procedure REFRESH:

then

end

Other: repeat forever

repeat

then

end

then

else

end
REFRESH

for $m:=1$ to $n o \_s o n s$ do ba_color $[m]:=\operatorname{read}\left(s_{b a}[m]\right)$;

if (all_out_links_balanced)

CRITICAL SECTION

if border $=0$

then

new_color $:=1-n e w \_c o l o r$

border $:=1$

end

unbalance_border_link

REFRESH

$\left\langle u b \_c o l o r\right.$, close $>:=\operatorname{read}\left(q_{u b}\right)$

until close $=0$

for $m:=1$ to $n o \_s o n s$ do $b a \_c o l o r[m]:=\operatorname{read}\left(s_{b a}[m]\right)$

if (all_out_links_balanced) and (in_link_unbalanced)

CRITICAL SECTION

if border $=0$

then

$r_{b a}:=$ write $\left(n e w \_c o l o r\right)$

new_color $:=1-n e w \_c o l o r$

border $:=1$

else

unbalance_border_link

end

Procedure unbalance_border_link

$r_{u b}[$ border $]:=\mathbf{w r i t e}(<1-$ new_color, $1>)$

ba_color $[$ border $]:=\operatorname{read}\left(s_{b a}[\right.$ border $\left.]\right)$

if (border_link_balanced)

$r_{u b}[$ border $]:=$ write $(<$ new_color, 0$\left.\rangle\right)$

border $:=$ border +1 mod (no_sons +1$)$

$r_{u b}[$ border $]:=$ write $(<1-$ new_color, $0>)$

for $m:=1$ to border -1 do $r_{u b}[m]:=$ write $(\langle$ new_color, 0$\rangle)$

for $m:=$ border to no_sons do $r_{u b}[m]:=$ write $\left(\left\langle 1-n e w \_c o l o r, 0\right\rangle\right)$

if $n o t \_r o o t$ then $r_{b a}:=$ write $\left(1-n e w \_c o l o r\right)$

$\{$ refresh register values\}

Figure 4.2: The Mutual-Exclusion Protocol for Dynamic Tree Systems. 
The code of the protocol, for the root and for a normal processor appears in Figure 4.2. The program for a processor is a loop which is executed forever. Analogous to the balance-unbalance protocol the loop is divided to a refresh block and a main block. The refresh block consists a subroutine called REFRESH in which all the processor's registers are refreshed. After REFRESH is executed entirely once, the processor is refreshed and the colors in all its registers can be deduced by the values of border and new_color.

After a processor is refreshed it proceeds to execute its main block. Processor $P$ starts its main block by reading all the balance registers of its sons. In addition a non-root processor reads the unbalance register of its father and waits until its incoming link is open. Analogous to the balance-unbalance protocol a processor proceeds to execute its main block only if it is privileged. A processor checks that it is privileged by use of two predicates called all_out_links_balanced and in_link_unbalanced (the root only checks predicate all_out_links_balanced). Both predicates are checked using local values only, no additional read actions are required. Predicate all_out_links_balanced holds if the processor's internal variables indicate that all its outgoing links are balanced. Predicate in_link_unbalanced holds if $u b \_c o l o r=1-n e w \_c o l o r$. Since the processor only checks its internal variables it may get an erroneous indication, in the correctness proof below we show that this situation happens only finitely many times.

In order to prove that the protocol is self-stabilizing we first define the set $M E$ of legitimate sequences of system configurations. Any sequence $s \in M E$ satisfies the following:

[Exclusion]: In each configuration $C$ of $s$ at most one processor is privileged.

[Fairness]: During $s$ each processor is privileged infinitely often.

A subtree $T$ is uniformly colored in some configuration $C$, if in $C$, all the processors in $T$ are refreshed, all registers of $T$ have the same color and all links of $T$ are open. To get some intuition on how the protocol stabilizes note that after a processor is refreshed, it is privileged only if all its outgoing links are balanced. When the processor is privileged it "assumes" that all its subtrees left of the border link are colored with new_color and that the border subtree itself and all the subtrees right of the border link are colored with the complementing color. When any of its outgoing links is not balanced the processor "waits"' until its son balances the link. As the execution proceeds larger subtrees become uniformly colored until the entire tree is uniformly colored. In the sequel we prove that any configuration in which the entire tree is uniformly colored is a safe configuration for the protocol. 
Lemma 4.1: Let $E$ be an arbitrary fair execution. If during $E$ the colors of all registers in the system are constant then there exists a configuration $C_{t}$ in $E$ such that for every subsequent configuration $C_{u}(u \geq t)$ all the system links are open.

Proof: We prove the lemma by showing that for every processor $P_{i}$ in the system there is an index $t(i)(t(i)$ depends on $E$ ) such that starting in $C_{t(i)}$ and subsequently throughout $E$ all the outgoing links of $P_{i}$ are open. The proof proceeds by induction on $d$, the distance of $P_{i}$ from the root.

Base Case: $d=0$. In this case $P_{i}$ is the root processor. Since $E$ is fair the root refreshes its registers infinitely often. Let $C_{t(i)}$ be the configuration reached by the system after the root executes REFRESH entirely for the first time. (a processor may start in the middle of REFRESH, in this case we only consider the second time in which REFRESH is executed.) In $C_{t(i)}$ all the root's outgoing links are open. Following $C_{t(i)}$ the root reads all the balance registers of its sons. Since in $E$ all colors are constant, by the time this read step is over the root has a correct view. The proof proceeds now by assuming that the root closes a link. Applying Lemma 3.1 to show that whenever the root closes a link it subsequently changes the color of the link's unbalance register, contradiction.

Induction Step: We assume correctness of the lemma for all processors at distance $d$ from the root. Let $P_{i}$ be an arbitrary processor at distance $d+1$ from the root. We show the existence of a configuration $C_{t(i)}$ after which all $P_{i}$ 's outgoing links are open. Let $P_{f}$ be the "father" of $P_{i}$. The distance of $P_{f}$ from the root is $d$. By the induction hypothesis there exists a configuration $C_{t(f)}$ after which $P_{i}$ 's incoming link is open throughout $E$. Therefore following $C_{t(f)} P_{i}$ 's behavior is similar to the root's behavior and the same proof applies.

Lemma 4.2: Eventually, the color of at least one register in the system is changed.

Proof: Assume towards a contradiction that $E$ is a fair execution during which no processor changes the color of any of its registers. By Lemma 4.1 there exists a configuration $C_{t}(t \geq 0)$ in $E$ such that for every configuration $C_{u}(u \geq t)$ all the system links are open.

Case 1: In $C_{t}$ all the links of the root are balanced.

Following $C_{t}$ and after the root is refreshed, it reads the balance registers of all its sons, discovers that it is privileged and subsequently changes the color of its border link, contradiction. 
Case 2: In $C_{t}$ at least one of the root's outgoing links is not balanced.

By the assumption no color field is changed. Hence any unbalanced (balanced) link in $C_{t}$ remains unbalanced (balanced) during $E$. Consider an unbalanced link $\left(P_{f} \rightarrow P_{i}\right)$ of maximal distance from the root. The incoming link of $P_{i}$ is unbalanced and all the outgoing links of $P_{i}$ are balanced in any configuration of $E$. Following $C_{t}$, $P_{i}$ 's behavior is similar to the root's behavior and the proof for case 1 applies.

Corollary 4.3: In every fair execution the color of at least one register is changed infinitely often.

Proof : The proof is immediate by a repeated application of Lemma 4.2.

\section{Lemma 4.4:}

(a) If a processor $P$ changes the color of one of its registers infinitely often, then $P$ executes its loop infinitely often.

(b) Once a processor $P$ executes its loop entirely, $P$ is refreshed.

Proof: (a) follows by the observation that during one execution of its loop, $P$ may change the color of each of its registers at most twice (at most once after the first time $P$ completes its loop). (b) is implied immediately by (a) since REFRESH is part of P's loop.

Lemma 4.5: Let $P$ be an arbitrary processor, let $e_{1}$ be $P$ 's incoming link and let $e_{2}$ be an arbitrary outgoing link of

$P$. Let $r_{u b}^{1}$ and $r_{u b}^{2}$ be the unbalance registers of $e_{1}$ and $e_{2}$ respectively, and let $r_{b a}^{1}$ and $r_{b a}^{2}$ be the balance registers of $e_{1}$ and $e_{2}$ respectively. The following claims hold:

(a) If the color of $r_{b a}^{1}$ is changed infinitely often, so is the color of $r_{u b}^{2}$.

(b) If the color of $r_{b a}^{1}$ is changed infinitely often, so is the color of $r_{u b}^{1}$.

(c) If the color of $r_{u b}^{2}$ is changed infinitely often, so is the color of $r_{b a}^{2}$.

(d) If the color of $r_{u b}^{2}$ is changed infinitely often, so is the color of $r_{b a}^{1}$.

Proof: By Lemma 4.4 each of the conditions in items a-d implies that $P$ executes its loop infinitely often and in particular $P$ is eventually refreshed in $E$.

(a) Once $P$ is refreshed and throughout $E$ it holds that right before the color of $r_{b a}^{1}$ changes, the color of $r_{b a}^{1}$ is not equal to the color of $r_{u b}^{2}$. This last assertion implies that between any two successive changes of the color of $r_{b a}^{1}$, the color of $r_{u b}^{2}$ is changed too. 
(b) If $r_{b a}^{1}$ is changed infinitely often, then eventually it is changed only after $P$ executes line 7 and finds that the incoming link $e_{1}$ is unbalanced. Assume for contradiction that $r_{u b}^{1}$ is never changed from some point on. After this point, whenever $P$ writes to $r_{b a}^{1}$ the link becomes balanced, and it remains so until the next time $P$ checks the condition in line 7 . Since the link remains balanced, this condition does not hold. Hence, $P$ never changes $r_{b a}^{1}$ again, contradiction.

(c) The proof here is similar to the proof of (b).

(d) The proof is identical to the proof of (a).

Lemma 4.6: In every fair execution of the protocol the color of every register is changed infinitely often.

Proof: By Corollary 4.3, the color of some register is changed infinitely often. By a repeated application of (b) and (d) of Lemma 4.5, this implies that the color of a register of the root processor is changed infinitely often. Assume that the root has no_sons sons. Whenever the root colors a register, the value of border is increased by 1 (mod no_sons). This means that the color of all the root's registers is changed infinitely often. The proof is now completed by a repeated application of (a) and (c) of Lemma 4.5 .

Since the color of every register in the system changes infinitely often we can now use Lemma 3.5. For this we consider a fair execution of the tree protocol, $E$, and regard the color changes on the registers of any link during $E$, as a separate execution of the balance-unbalance protocol. Using this method we get:

Corollary 4.7 In every fair execution every link descriptor reaches a configuration in the legitimate cycle (of Figure $3.3)$.

We proceed by combining the above results in order to show that eventually the system converges to a configuration in which the entire system tree is uniformly colored.

Lemma 4.8: There are configurations in $E$ in which the entire system tree is uniformly colored.

Proof: Let $E^{\prime}$ be a suffix of $E$ in which all registers are refreshed and all link descriptors follow the legitimate cycle. Consider an arbitrary link $e=\left(P_{f} \rightarrow P_{s}\right)$ : In $E^{\prime}$ the predicate all_out_links_balanced holds for $P_{s}$ only when all its outgoing links are indeed balanced. Thus in $E^{\prime}$, after $e$ is unbalanced, $P_{s}$ rebalances $e$ (by changing the color of its balance register) only when $e$ is open and after $P_{s}$ 's outgoing links are balanced and colored by the color of the unbalance register of $e$. Using this fact, we prove the following: 
Claim: Eventually, for each link $e=\left(P_{f} \rightarrow P_{s}\right), P_{s}$ writes 0 in the balance register of $e$ only when the subtree rooted at $P_{s}$ is uniformly colored by 0.

Proof of claim: The proof is by induction on $h$, the height of the subtree rooted at $P_{s}$. The claim holds trivially if $P_{s}$ is a leaf. Assume it holds for any processor $P$ such that the height of the subtree rooted at $P$ is smaller then $h$. Let the height of the subtree rooted at $P_{s}$ be $h$. Consider a configuration in which $P_{f}$ writes 0 into the unbalance register of $e$, setting the link descriptor to $(1,0,0->1,1)$. It was already proved that $P_{s}$ rebalances the link only after the registers of all its outgoing links are balanced and colored 0. Since the link descriptors are changed according to the legitimate cycle, those outgoing links are balanced by the sons of $P_{s}$. By the induction hypothesis, each son of $P_{s}$ rebalances its incoming link only after its subtree is informally colored with 0 , hence when all links outgoing from $P_{s}$ are balanced with 0 the entire subtree rooted at $P_{s}$ is colored with 0 . This proves the claim.

The proof of the lemma is completed by applying the claim to the links emanating from the root processor. For this, consider a configuration $C$ in which all the root registers are colored 0 (there are infinitely many such configurations in $E$ - see proof of Lemma 4.6), and all the link descriptors are changed according to the legitimate cycle. There is a configuration $C^{\prime}$ following $C$ in which all the root's outgoing links are balanced to 0 (otherwise the root never colors any of its registers). But $C^{\prime}$ could be reached only by having all the root sons balancing their incoming links to 0 , which by the claim means that all the subtrees rooted at the root's sons are uniformly colored by 0 , and hence the entire tree is uniformly colored.

We proceed by showing that eventually, in any configuration at most one processor is privileged.

Lemma 4.9: Eventually there is a single privileged processor in every configuration.

Proof: Recall that a privileged processor in our protocol is a processor $P$ which is about to execute either line 3 or line 7 of the code and for which the predicates in_link_unbalanced and all_out_link_balanced are true. Once every link descriptor is in the legitimate cycle, those predicates are true for $P$ only if indeed the incoming link of $P$ is unbalanced and its outgoing links are balanced. To prove the lemma it suffices to prove that eventually there is at most one processor for which the incoming link is unbalanced (or it is the root) and all its outgoing links are unbalanced. We will prove the following stronger result:

Claim: Eventually, in every configuration there is at most one processor, say $P$, whose incoming link is unbalanced (or it is the root), and all its outgoing link are balanced. Moreover, all the links in the system are balanced, except the 
links on the path from the root to $P$.

Proof of Claim: By induction on the order of configurations in $E$. The induction base is a configuration $C_{t}$ in which all registers and variables are colored 0. By Lemma 4.8 this configuration, which satisfies the claim, is reached in every fair execution. Assume that the claim holds for a certain configuration $C_{u}(u \geq t)$. We have to show that the claim holds for $C_{u+1}$.

Let $P$ be the only privileged processor in $C_{u}$. If the atomic step between $C_{u}$ and $C_{u+1}$ does not change the value of any register then we are done. If the atomic step changes the value of some register say $r$, then $r$ is a register of $P$ (since $P$ is the only privileged processor, and only a privileged processor may write). The fact that every link descriptor is in the legitimate cycle, implies that if $r$ is the unbalance register of an outgoing link then $P$ unbalances the link, and the claim holds for $P$ 's son. If $r$ is the balance register of $P$ 's incoming link, then $P$ balances the link, and the claim holds for $P$ 's father. This completes the proof of the claim, and hence of the Lemma.

Corollary 4.10: The protocol is self-stabilizing relative to the set $M E$.

Proof: By Lemma 4.9 at most one privileged processor exist hence the [exclusion] requirement holds. By Lemma 4.6 each processor is privileged infinitely often hence the [fairness] requirement holds.

\section{A Mutual-Exclusion Protocol for Dynamic Networks}

In this section we present a mutual exclusion protocol for dynamic networks. Consider a semi-uniform system in which each link is augmented by two read only registers called the tree-registers. The tree registers encode a spanning tree of the communication graph rooted at the special processor as follows: The registers for each link should specify whether the link belongs to the spanning tree; in case the link belongs to the spanning tree its registers should specify its direction; these parameters can be obtained by the processors on the link's endpoints. In this case a selfstabilizing, mutual exclusion protocol for a system with any (static) communication graph can be derived simply by pre-computing a spanning tree for this system's communication graph and encoding it into the tree registers. The tree registers can be hardwired and therefore constant throughout any execution. The protocol is obtained by executing the mutual exclusion protocol for tree systems where links which are not in the spanning tree are ignored.

This however falls short of our aim in this paper since the resulting protocol is not dynamic and it requires some pre-computing. On the other hand this protocol motivates the following ideas: 
(1) A rooted spanning tree of the communication graph will be computed by a self-stabilizing protocol whose registers are eventually constant.

(2) The registers of the spanning tree protocol will be used as tree registers for the mutual exclusion protocol.

(3) Both protocols will be combined to achieve a dynamic, self-stabilizing, semi-uniform protocol for mutual exclusion on general graphs.

In the rest of this section we show how these ideas are implemented.

5.1. A Rooted Spanning Tree Protocol. The spanning tree protocol produces a BFS tree of the system's communication graph. Let $G(V, E)$ be a graph with orderings $\alpha=\left(\alpha_{1}, \alpha_{2}, \cdots \alpha_{n}\right)$ of the edges incident to each node $v_{i} \in V$. Define the First BFS Tree of $G$ relative to $v_{1}$ and $\alpha$ to be a BFS tree, rooted at $v_{1}$. In case a node, $v_{i}$ of distance $d+1$ from $v_{1}$ has more than a single neighbor of distance $d$ from $v_{1}, v_{i}$ is connected to its first neighbor, according to $\alpha_{i}$, whose distance from $v_{1}$ is $d$. The protocol always produces the First BFS Tree of the system's communication graph, with respect to the node of the special processor and to the (arbitrary) orderings $\alpha=\left(\alpha_{1}, \alpha_{2}, \cdots \alpha_{n}\right)$ in which the neighbors of all processors are ordered. The special processor is called the root processor.

Essentially the protocol is a distributed $B F S$ protocol. Each processor is continuously trying to compute its distance from the root and report it to all its neighbors by writing it in its registers. At the beginning of an arbitrary execution the only processor which is guaranteed to compute the right distance is the root itself, once this distance is written in all the root's registers, the value stored in these registers will never change. Once all processors of distance $d$ from the root have completed to compute their distance from the root correctly and write it in all their registers, their registers remain constant throughout the execution and processors of distance $d+1$ from the root are ready to compute their own distance from the root and so on and so forth. The spanning tree protocol bears some similarity to the ARPANET routing protocol [Ta77,Ta81] (that latter protocol assumes a different model).

The output tree is encoded by means of the registers as follows: Each register $r_{i j}$, in which $P_{i}$ writes and from which $P_{j}$ reads, contains a binary father field denoted by $r_{i j}$ father. If $P_{j}$ is the father of $P_{i}$ in the output $B F S$ tree then the value of $r_{i j}$ father is 1 , otherwise the value of $r_{i j}$ father is 0 . In addition each register $r_{i j}$ has a distance field, denoted by $r_{i j}$.distance which holds the distance from the root to $P_{i}$. 
The code of the protocol, for the root and for the other processors, appears in Figure 5.1. In this code the number of the processor's neighbors is given by the parameter no_neighbors. The program for the root is very simple: It keeps "telling" all its neighbors that it is the root by repeatedly writing the values $\langle 0,0\rangle$ in all its registers. The first 0 is the distance from the root to itself, the second 0 tells each neighbor that it is not the father of the root. The program for a normal processor consists of a single loop. In this loop the processor reads all the registers of its neighbors. Processor $P_{i}$ which has no_neighbors neighbors keeps no_neighbors internal variables corresponding to the no_neighbors registers from which $P_{i}$ reads. The internal variable, corresponding to register $r_{j i}$ is denoted by $i r_{j i}$. This variable stores the last value of $r_{j i}$ read by $P_{i}$. Its two fields are denoted by $i r_{j i}$. father and $i r_{j i}$.distance respectively. Once all these registers are read, $P_{i}$ computes a value for the variable dist which represents $P_{i}$ 's current idea of its distance from the root. The purpose of the boolean variable first_found is to make sure by the end of each pass of the loop that each processor has a single father. The minimum in line $\left(^{*}\right)$ is taken over $m$, $1 \leq m \leq$ no_neighbors.

The task $S T$ of legitimate sequences is defined as the set of all configuration sequences in which every configuration encodes the first $B F S$ tree of the communication graph. In the following lemma we characterize the set of safe configurations for the protocol:

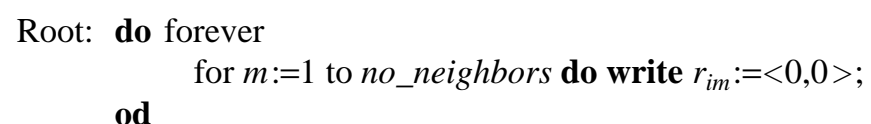

Other: do forever

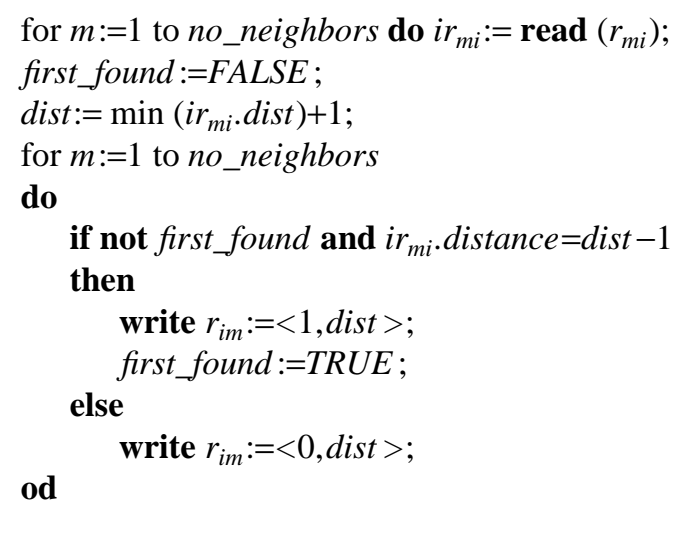

Figure 5.1: The Spanning Tree Protocol for $P_{i}$. 
Lemma 5.1: Let $c$ be a system configuration which satisfies:

(a) The processors registers encode the first BFS tree of the system's communication graph rooted at the root.

(b) For each normal processor $P_{i}$ and for each of its neighbors $P_{j}, i r_{i j}=r_{i j}$.

(c) For each normal processor $P_{i}$, the local variable dist stores the distance of $P_{i}$ from the root and the value of the local variable first_found satisfies: If the value of the local variable $m$ is $m_{0}$ and $P_{i}$ has a neighbor of distance dist-1 from the root whose rank according to $\alpha_{i}$ i $<m_{0}$ then first_found=TRUE, otherwise first_found $=F A L S E$.

Configuration $c$ is safe for the protocol.

Proof: It is not hard to verify that following $c$ the value of no register ever changes hence all subsequent configurations encode the first $B F S$ tree of the system's communication graph as required by the definition of the set $S T$.

The following lemma shows that in every execution a safe configuration is reached.

Lemma 5.2: Let $P_{i}$ be an arbitrary processor whose distance from the root is $l$ and let $P_{j}$ be an arbitrary neighbor of $P_{i}$. Let $E=c_{0}, c_{1}, \cdots$ be an arbitrary fair execution of the protocol. For every integer $d \geq 0$ there exist $t_{d}$ such that: For every integer $t, t \geq t_{d}$, the configuration $c_{t}$ satisfies the following assertions:

(a) $\quad$ If $l \leq d$ then $r_{i j}$.distance $=l$.

(b) If $l \leq d$ then $r_{i j}$ father has the "right" value. That is: if $P_{j}$ is the first neighbor of $P_{i}$ (using $\alpha_{i}$ ) of distance $l-1$ from the root then $r_{i j}$ father $=1$, and otherwise $r_{i j}$ father $=0$.

(c) If $l<d$ then $i r_{j i}=r_{j i}$.

(d) If $l>d$ then $r_{i j}$.distance $>d$.

Proof: We prove the theorem by induction over $d$. In the proof we use the fact that due to the fairness of $E$ every processor is activated in $E$ infinitely often.

Base Case: $(d=0)$ The only node of distance 0 from the root is the root itself. Assume that the root has no_neighbors neighbors. After no_neighbors activations of the root, all its registers store the value $\langle 0,0\rangle$. The values stored in the registers of the root will not be changed any more. This completes the proof of assertion (a). Assertion (b) is also satisfied since no processor is the "father" of the root. Assertion (c) holds vacuously for the base 
case, since there are no processors of distance $<0$ from the root. Assertion (d) is trivially satisfied since the permitted values of the distance field are the natural numbers which are all positive.

Induction Step: $(d>0)$ Let $t_{d}$ be an integer such that for every $t \geq t_{d}$, configuration $c_{t}$ satisfies assertions (a) $-(\mathrm{d})$ for some integer $d, d>0$. We show the existence of some integer $t_{d+1}$ such that for every integer $t \geq t_{d+1}$ configuration $c_{t}$ satisfies assertions (a) $-(\mathrm{d})$ for $d+1$.

If the distance of $P_{i}$ from the root is $d+1$ then all its neighbors are of distance $\geq d$ from the root. Moreover $P_{i}$ has at least one neighbor, whose distance from the root is exactly $d$. By assertion (a) of the induction hypothesis, for every $P_{k}$ of distance $d$ from the root, it holds that the value stored in $r_{k i}$.distance in $c_{t_{d}}$ and all subsequent configurations is $d$. By assertion (d) of the induction hypothesis, for every $P_{l}$ of distance $>d$ from the root, it holds that the value stored in $r_{l i}$.distance in $c_{t_{d}}$ and all subsequent configurations is $>d$. Therefore, whenever $P_{i}$ executes line (*) after $c_{t_{d}}$, the value assigned to the variable dist is exactly $d+1$. Once this value is written in all registers of $P_{i}$, assertions (a) and (b) hold for $P_{i}$. The same holds for all processors of distance $d+1$ from the root. Hence there is a configuration $c_{1}$ reached by the system, such that for every configuration $c$ following $c_{1}$, assertions (a) and (b) are satisfied for all processors of distance $d+1$ from the root.

It is easy to see that from $c_{1}$ and onwards forever, the values stored in the registers of all processors of distance $d+1$ from the root will not be changed any more. In particular all neighbors of all processors of distance $d$ from the root will not change the values stored in their registers any more. If $P_{i}$ is a processor of distance $<d+1$ from the root then each read action after $c_{1}$ sets one of its internal variables to its final stationary value. Thus there is a configuration $c_{2}$ reached by the system, such that every configuration $c$ following $c_{2}$ satisfies assertion (c) for $d+1$.

Let $P_{i}$ be an arbitrary processor of distance $>d+1$ from the root. The neighbors of $P_{i}$ are all of distance $\geq d+1$ from the root. By assertion (d) of the induction hypothesis starting from $c_{t_{d}}$ and onwards each neighbor $P_{j}$ of $P_{i}$ satisfies $r_{j i}$.distance $>d$. Therefore, whenever $P_{i}$ executes line $\left(^{*}\right)$ after $c_{t_{d}}$ the value assigned to the variable dist is $>d+1$. Once this value written to all registers of $P_{i}$ assertion (d) is satisfied for $P_{i}$. The same holds for all processors of distance $>d+1$ from the root. Hence there is a configuration $c_{3}$ reached by the system, such that every configuration following it satisfies assertion (d) for $d+1$. Let $c_{t_{d+1}}$ be the later configuration among $c_{2}$ and $c_{3}$. It is easy to see that indeed every configuration $c$ following $c_{t_{d+1}}$ satisfies assertions (a)-(d) for $d+1$. 
Corollary 5.3: The protocol presented above is self-stabilizing relative to the set $S T$.

5.2. Fair Combination of Self Stabilizing Protocols. A self-stabilizing mutual exclusion protocol for general graphs can be obtained by combining the self-stabilizing rooted spanning tree protocol presented in subsection 5.1 with the self-stabilizing mutual exclusion protocol for dynamic tree-structured systems, presented in section 4 , as follows: The combined protocol runs both protocols alternately, such that the later protocol uses the tree encoded by the tree registers written by the former protocol. By the correctness of the spanning-tree protocol, the tree registers eventually encode a spanning tree, and are subsequently constant throughout the execution. By the correctness of the mutual exclusion protocol, it eventually converges to a legitimate execution of mutual exclusion on this spanning tree, and hence on the entire graph.

We now formalize and extend this idea to a general technique of fair protocol combination. In this technique two simple protocols, called a "slave", protocol and a "master" protocol, are combined to obtain a more complex protocol. In the formal definition we assume that both protocols are shared memory protocols but we do not impose any restriction on either the exact model (register or non-register) or on the specific communication graph or on the protocols' atomicity level. Using proper definitions can also eliminate the shared memory assumption.

Assume that the "slave" protocol is called $P r_{1}$, for a task $T_{1}$ and that the "master" protocol is called $P r_{2}$ for a task $T_{2}$. The state set of a processor $P_{i}$ in the combined protocol is $S_{i}=A_{i} \times B_{i}$, where $A_{i}$ is the state set of $\operatorname{Pr}_{1}$ and $A_{i} \times B_{i}$ is the state set of $\operatorname{Pr}_{2}$ but we assume that $\operatorname{Pr}_{2}$ modifies only the $B_{i}$ components. The state transition function of the slave protocol $\operatorname{Pr}_{1}$ for processor $P_{i}$ is a function $f: A_{i} \rightarrow A_{i}$, while the state transition of the master protocol $\operatorname{Pr}_{2}$ for $P_{i}$ is a function $g: A_{i} \times B_{i} \rightarrow B_{i}$. These transition functions are extended to functions over $S_{i}$ as follows: For $(a, b) \in S, f((a, b))$ is $(f(a), b)$ and $g((a, b))$ is $(a, g(a, b))$. In the combined mutual-exclusion protocol, $\operatorname{Pr}_{1}$ is the spanning tree protocol and the $A_{i}$ 's are the states modified by this protocol, including the tree registers; $\operatorname{Pr}_{2}$ is the version of the mutual exclusion protocol which uses the tree registers to encode the tree edges on which it operates.

The next definitions formalizes the concept of an execution of the master protocol which assumes a selfstabilized execution of the slave protocol. Let $S_{i}, A_{i}, B_{i}$ and $\operatorname{Pr}_{2}$ be as above, and let $T_{1}$ be a task in which the states of processor $P_{i}$ are in $A_{i}$. Assume that $T_{1}$ is closed under stuttering (i.e., for each sequence $L$ in $T_{1}$, the sequence obtained from $L$ by duplicating each entry finitely many times is also in $T_{1}$ ). For configuration $C, C \in S_{1} \times \cdots \times S_{n}$ define the $A$-projection of $C$ as the configuration $\left(a_{1}, \cdots, a_{n}\right) \in A_{1} \times \cdots \times A_{n}$. For a sequence of configurations 
$L=\left(C_{1}, C_{2}, \cdots\right)$, the $A$-projection of $L$ is the sequence $\left(A_{1}, A_{2}, \cdots\right)$, where $A_{i}$ is the $A$-projection of $C_{i}$.

A fair execution of $\operatorname{Pr}_{2}$ given $T_{1}$ is a sequence of configurations $E=\left(C_{1}, C_{2}, \cdots\right)$ such that

(a) For every two consecutive configurations $C_{i}=\left(A_{i}, B_{i}\right)$ and $C_{i+1}=\left(A_{i+1}, B_{i+1}\right)$, either $A_{i}=A_{i+1}$ or $B_{i}=B_{i+1}$.

(b) If $B_{i} \neq B_{i+1}$ then the transition from $C_{i}$ to $C_{i+1}$ is a transition of $P r_{2}$, and the sequence of these transitions is fair (i.e., each processor is activated in it infinitely often).

(c) The A-projection of $E$ belongs to $T_{1}$.

Condition (b) says that the modifications of the states in the $B_{i}$ 's are done by $\operatorname{Pr}_{2}$ in a fair manner, while condition (c) says that the sequence of states in the $A_{i}$ 's forms a legitimate sequence of task $T_{1}$.

We say that protocol $\mathrm{Pr}_{2}$ is self-stabilizing for task $T_{2}$ given task $T_{1}$ if any fair execution of $\mathrm{Pr}_{2}$ given $T_{1}$ has a suffix in $T_{2}$. Finally, a protocol $P r$ is a fair combination of $P r_{1}$ and $P r_{2}$ if in $\operatorname{Pr}$ every processor executes steps of $\mathrm{Pr}_{1}$ and $\mathrm{Pr}_{2}$ alternately. Note that for an execution $E$ of $\mathrm{Pr}$, the $A$-projection of $E$ is a sub-execution of $E$ corresponding to a fair execution of the slave protocol $\operatorname{Pr}_{1}$.

The following theorem gives sufficient conditions underwhich the composition of two self-stabilizing protocols is also self-stabilizing:

Theorem 5.4: Assume that $P r_{2}$ is self-stabilizing for a task $T_{2}$ given task $T_{1}$. If $\operatorname{Pr}_{1}$ is self stabilizing for $T_{1}$, then the fair combination of $\operatorname{Pr}_{1}$ and $\operatorname{Pr}_{2}$ is self-stabilizing for $T_{2}$.

Proof: Consider any execution $E$ of $P r$, the fair combination of $P r_{1}$ and $P r_{2}$. By the self-stabilization of $\operatorname{Pr}_{1}, E$ has a suffix $E^{\prime}$ such that the $A$-projection of $E^{\prime}$ is in $T_{1}$. By the assumption that $P r_{2}$ is self-stabilizing given $T_{1}, E^{\prime}$ has a suffix in $T_{2}$.

Theorem 5.4 provides a general methodology to construct self-stabilizing protocols for complex tasks: Given a task $T_{2}$ for which we wish to construct the protocol, first define a task $T_{1}$ and construct a protocol $P r_{2}$ which is self stabilizing for $T_{2}$ given $T_{1}$, and then construct a protocol $P r_{1}$ which is self-stabilizing for $T_{1}$. The fair combination of $\operatorname{Pr}_{1}$ and $P r_{2}$ is the desired protocol. Note that this methodology does not require that the protocol $P r_{1}$ reaches a "steady state", in which the communication registers (or any other component in the state $a_{i}$ of processor $P_{i}$ ) are never changed. 
Corollary 5.5: The fair composition of the spanning tree protocol with the mutual exclusion protocol is a mutual exclusion protocol on systems with an arbitrary dynamic communication graph. This protocol is self-stabilizing in the presence of the distributed demon under read/write atomicity.

We conclude this section by observing that the notion of fair combination of protocols can be further extended, by allowing the protocol $\operatorname{Pr}$ to interleave the executions of the protocols $P r_{1}$ and $P r_{2}$ in an arbitrary way, and not necessarily in alternating manner. In this more general setting, each processor switches from executing protocol $P r_{1}$ to executing protocol $\mathrm{Pr}_{2}$ and vice versa according to some internal conditions, which should guarantee fair execution of both protocols. This adds extra flexibility to the way by which one can achieve composite protocols by combining simpler ones. We demonstrate this by the following general theorem, concerning the fair combination our mutual exclusion protocol with any arbitrary self-stabilizing protocol. In this theorem we assume the register model used in the previous sections.

Theorem 5.6: For any semi-uniform protocol $\mathrm{Pr}_{2}$ which is self-stabilizing under composite atomicity there is a semi-uniform protocol $\mathrm{Pr}$ which is self-stabilizing (for the same task) under read/write atomicity.

Proof: We describe a protocol $\operatorname{Pr}$ which simulates $\operatorname{Pr}_{2}$ under read/write atomicity. $\mathrm{Pr}$ is a fair composition of $\operatorname{Pr}_{2}$ as the master protocol and the mutual exclusion protocol presented above, as the slave protocol $\operatorname{Pr}_{1}$. We describe $\operatorname{Pr}$ by describing the rules by which it switches from executing $\operatorname{Pr}_{2}$ to executing $\operatorname{Pr}_{1}$ and vice-versa.

Each state transition of $\mathrm{Pr}_{2}$ under composite atomicity can be written as a sequence of atomic steps under read/write atomicity. Whenever a processor $P_{i}$ is scheduled to operate, it first checks if it is in critical section according to $P r_{1}$. If not, then $P_{i}$ executes a step of $P r_{1}$. If $P_{i}$ enters its critical section in $P r_{1}$, it stops executing $\operatorname{Pr}_{1}$ and executes steps of $\mathrm{Pr}_{2}$, until it completes one state transition of $\mathrm{Pr}_{2}$ under the composite atomicity (this may take many atomic steps under the read/write atomicity). Once this is done, $P_{i}$ transfers the privilege to one of its neighbors, according to $\operatorname{Pr}_{1}$, and so on and so forth. The mutual exclusion property ensures that as long as $P_{i}$ does not complete its state transition in $\mathrm{Pr}_{2}$, no other processor (and in particular no neighbor of $P_{i}$ ) executes any state transition of its own. The fair combination ensures that each processor enters its critical section infinitely often. Thus, each execution of $\mathrm{Pr}$ has a suffix which is equivalent to a fair execution of $\mathrm{Pr}_{2}$ under composite atomicity. 


\section{Concluding Remarks}

A semi-uniform, dynamic, self-stabilizing, mutual-exclusion protocol for systems with an arbitrary communication graph was presented. The protocol is correct in the presence of read/write atomicity under the distributed demon. (For protocols that use read/write atomicity the distributed demon and the central demon are equivalent) Using this protocol we showed that any self-stabilizing protocol which is correct under composite atomicity can be executed under read/write atomicity in a self-stabilizing fashion.

Although this paper does not concern itself with complexity measures it is worth mentioning that when time is measured by some appropriately defined round complexity, the stabilization time of the mutual exclusion protocol is

$O\left(n^{2}\right)$. The stabilization time of the spanning tree protocol is $O(D)$, where $D$ is the diameter of the system's communication graph.

\section{Acknowledgment:}

We thank three anonymous referees for their constructive remarks on an earlier version of this paper. The help of Frank Stomp is also appreciated. 


\section{REFERENCES}

[BGW87] G. M. Brown, M.G. Gouda, and C. L. Wu: A Self-Stabilizing Token System, Proc. of the Twentieth Annual Hawaii International Conference on System sciences, 1987, pp. 218-223.

[Bu87] J. E. Burns: Self-Stabilizing Rings without Demons, Technical Report GIT-ICS-87/36, Georgia Institute Of Technology, 1987.

[BP89] J. E. Burns and J. Pachl: Uniform Self-Stabilizing Rings, ACM Transactions on Programming Languages and Systems 1 (2) (1989), pp. 330-344.

[Dij74] E. W. Dijkstra: Self-Stabilizing Systems in spite of Distributed Control, Communications of the ACM 17, no. 11 (1974), pp. 643-644.

[Dij82] E. W. Dijkstra: Self-Stabilizing Systems in spite of Distributed Control (EWD391), Reprinted in Selected Writing on Computing: A Personal Perspective, Springer-Verlag, Berlin, 1982, pp. 41-46.

[Dij86] E. W. Dijkstra: A Belated Proof of Self-Stabilization, Distributed Computing 1, 1 (1986), pp. 5-6.

[DIM89] S. Dolev, A. Israeli and S. Moran: preliminary version of "Self-Stabilization of Dynamic Systems Assuming only Read/Write Atomicity," Proc. MCC Workshop on Self-Stabilization, Austin, Texas, November 1989. Also in Proceedings of the 9th Annual ACM Symposium on Principles of Distributed Computing, August 1990, pp. 103-117.

[IJ90] A. Israeli and M. Jalfon: Token Management Schemes and Random Walks Yield Self-Stabilizing Mutual Exclusion, Proceedings of the 9th Annual ACM Symposium on Principles of Distributed Computing, August 1990, pp. 119-131.

[IJ91] A. Israeli and M. Jalfon: Uniform Self-Stabilizing Ring Orientation, To be published in Information and Computation. Also in Lecture Notes in Computer Science 486: Distributed Algorithms (Proceedings of the Fourth International Workshop on Distributed Algorithms, Bari, Italy, September 1990), J. Van Leeuwen and N. Santoro, Editors, pp. 1-14, Springer-Verlag, 1991 
[KP89] S. Katz and K. J. Perry: Self-Stabilizing Extensions for Message-Passing Systems, this issue. Also in Proceedings of the 9th Annual ACM Symposium on Principles of Distributed Computing, August 1990, pp. 91-101.

[Kr79] H. S. M. Kruijer: Self-Stabilization (in spite of Distributed Control) in Tree-Structured Systems, Information Processing Letters 8,2 (1979), pp. 91-95.

[LA87] M. C. Loui and H. H. Abu-Amara: Memory Requirements for Agreement among Unreliable Asynchronous Processes, in Advances in Computing Research, JAI press, 1987, pp. 163-183.

[PF77] G. L. Peterson and M. J. Fischer: Economical Solutions for the Critical-Section Problem in a Distributed System, Proc. of ACM Symposium on Theory of Computing, 1977, pp. 91-97.

[Ta81] A. S. Tanenbaum: Computer Networks, Prentice-Hall, 1981, pp. 205-231.

[Ta77] W.P. Tajibhanapis: A Correctness Proof of a Topology Information Maintenance Protocol for a Distributed Computer Network, Communications of the ACM 20, no. 7 (1977), pp. 477-485.

[Tc81] M. Tchuente: Sur l'Auto-Stabilisation dans un R'eseau D'ordinateurs, RAIRO Inf. Theor. 15 (1981), pp. 47-66. 